kam es im September 1969 im Gebiet von Yendi, wobei 27 Menschen getötet und über 40 verletzt worden sein sollen.” (p. 11, note 2). The impression created here is that persons of different nationalities or tribes are involved in a minor war concerning chieftaincy. The obvious contradiction here seems to have escaped the author. Chieftaincy belongs to one nation or tribe and when there is any dispute concerning succession, it does not involve, directly, persons of other nations but fractions within the same nation. What the author has done here would be like describing struggles concerning the chancellorship in Germany as evidence of nationalism.

It is a pity that Goldmann ends his book on a topic which deserves more than the cursory treatment he offers, namely the language question in Ghana. As he correctly states, English remains the language of administration even after independence. Indeed, during the struggle for independence no voices were heard demanding the replacement of this foreign language by an African language. Here the author could have asked a few questions. Besides serving as an integrating factor, English serves as a tool for the domination of the masses by the elite. This explains to a great extent why nobody speaks against the use of a foreign language which is understood only by a tiny minority in the country. It is plain that the elite in Ghana, as in many other African countries, owe their position and prestige to their mastery of the English language and their acquisition or assimilation of English culture. Ability to use English can be used as a criterion for determining the class allegiance of many Ghanaians. Those who have imbibed English culture, with all its contempt for other languages, are hardly in a position to advocate its replacement by an African language. Indeed the idea has seldom occurred to the ruling elite.

Goldmann who is concerned with public administration could have asked himself how administrative policies made in English, and generally not translated into Ghanaian languages, are effectively implemented and at what cost. That injustice necessarily forms part of such a system needs hardly to be mentioned. How will a poor peasant with no idea of English be able to insist on his rights, and get justice from administrators who are generally contemptuous of him because of his background and inability to speak English? Mastery of English is the privilege of those selected few who have the chance to attend English-speaking schools in Ghana. An examination of the background of the civil servants (and incidentally of the lawyers, doctors, professors, etc.) would demonstrate beyond doubt the relationship of class and language in Ghana. When one considers that through the acquisition and use of a language certain definite world-views are transmitted, then the mutual re-inforcement of language, class, and ideology becomes obvious. But these are more the problems for the Ghanaian elite than for Dr. Goldmann whose detailed study offers outsiders an insight into problems of public administration in Ghana.

Kwame Opoku

\title{
Melvin Gurtov
}

China and Southeast Asia - The Politics of Survival

D. C. Heath and Co., Lexington, Mass., Toronto, London, 1971, xiii, 234 Seiten

Entsprechend der Analyse der Innenpolitik Chinas gibt es eine Art Wellentheorie der chinesischen Außenpolitik. Demzufolge folgte Peking nach der Machtkonsoli- 
dierung im Oktober 1949 einem linken Kurs. Mit der Beendigung der Kriege in Korea und im damaligen Indochina leitete China eine Politik der friedlichen Koexistenz mit neutralistischen oder freundlichen Ländern ein, die in der Bandung-Konferenz (April 1955) und danach zu einigen Erfolgen führte. Obwohl die Grenzauseinandersetzung mit Indien Ende der 50er Jahre u. a. einen Linksrutsch zu verkünden schien, unterstrich China durch Abkommen mit anderen Nachbarn seine Bereitschaft, Grenzstreitigkeiten auf friedliche Weise zu lösen. Die Kulturrevolution (1966-67) brachte Chinas Außenpolitik scheinbar wieder auf einen radikalen Kurs; durch eine Reihe von Zwischenfällen im In- und Ausland wurden die Beziehungen $\mathrm{zu}$ anderen Ländern getrübt oder gar abgebrochen. War dies ein Abschied von Bandung, eine neue linke Welle oder nur eine kurzfristige Verwirrung? Oder wurde Chinas Politik z. T. von Ereignissen in anderen Ländern provoziert?

In diesem Buch bekennt sich Melvin Gurtov, Associate Professor of Political Science, University of California, Riverside, zu der Auffassung, daß der außenpolitische Aufruhr zur Zeit der Kulturrevolution zum Teil durch Ereignisse im Ausland hervorgerufen wurde, zum Teil einer kurzfristigen Verwirrung in Peking zuzuschreiben sei, zu der die Besetzung des Außenministeriums durch Rotgardisten und der Ausschluß erfahrener Diplomaten aus der Außenpolitik beitrugen. In seiner Analyse der Beziehungen Chinas zu drei südostasiatischen Ländern, die als Modelfälle behandelt werden, betont er, wie sein Untertitel „A Study of Foreign Policy Interaction" verrät, die Interaktion nicht nur der Politik Chinas mit den anderen Ländern, sondern auch den Einfluß der amerikanischen und sowjetischen Initiativen, der Rivalität mit Indien und vor allem die Rolle des Konflikts im ehemaligen Indochina. Diese Aussage stützt der Verfasser durch die Analyse der Beziehungen zu zwei neutralistischen Ländern, Burma und Kambodscha (unter Sihanouk), und zu Thailand, einem Verbündeten der USA. Die Regierungen aller drei Länder standen einer internen kommunistischen Rebellion gegenüber, die möglicherweise von China unterstützt wurde. Behandelt wird hauptsächlich der Zeitraum von 1964, dem Beginn der Eskalation der amerikanischen Teilnahme am Vietnam-Krieg, bis 1970.

Hier sei nur auf die chinesische Seite eingegangen. Gurtov behauptet, daß Chinas Einstellung zu der Regierung in Bangkok, zur KP Thailands und zu den Rebellen im Nordosten durch Thailands Teilnahme an den Kriegen in Laos und Vietnam bedingt war. Als Thailand 1964-65 den Amerikanern Luftstützpunkte zur Verfügung stellte, somit von dort aus die Bombardierung nordvietnamesischer Stellungen ermöglichte, und später sogar Truppen nach Südvietnam sandte, ging China von der verbalen Unterstützung der KP und ihrer "Patriotischen Front" zu einem Aufruf zum Aufstand und Befreiungskrieg gegen die Regierung über. Peking geizte aber, so Gurtov, mit Geld- und Waffenhilfe in der UUberzeugung, daß die Rebellen schlecht organisiert und der Regierung unterlegen seien. Die chinesische Presse warnte die Aufständischen, daß ein „verlängerter" Krieg erforderlich sei, wenn auch der Endsieg durch Treue zu Mao Tse-tungs Denken gesichert sei. (Allerdings bekamen die Rebellen in Thailand den Löwenanteil ihrer Hilfe von Nordvietnam und nicht von Peking.) Würde Thailand seine engen Bindungen zur USA lockern und die Unterstützung Südvietnams aufgeben, so würde China die Unterstützung der Rebellen einschränken.

Kambodscha war für China hauptsächlich von symbolischer Bedeutung, da es bezeugte, daß neutralistische und unabhängige Länder gute Beziehungen zu China haben konnten. Sihanouk, auf der anderen Seite, hoffte auf Chinas Unterstützung in 
der Anerkennung seiner bedrohten Grenzen nach Thailand und Vietnam. Erst Ende Juli 1967, nach einer Anerkennungserklärung der Sowjetunion, gewann Sihanouk von Peking die gewünschte Anerkennung seiner Grenzen. Kurz danach erreichten Kambodschas Beziehungen zu China dank der kulturrevolutionären Entwicklung in Peking einen Tiefpunkt, ein Bruch aber wurde vermieden. März 1970, als das Problem der Versorgungslinien und Stützpunkte des Viet Cong und Nordvietnams auf Kambodschas Territorium zu Sihanouks Sturz führte (Gurtov glaubt, daß innenpolitische Auseinandersetzungen in erster Linie den Sturz verursachten und nicht etwa der amerikanische Geheimdienst), wartete Peking noch einige Wochen in der Hoffnung, daß das neue Lon Nol-Regime Sihanouks Neutralismus fortführen würde, bevor es den endgültigen Bruch besiegelte.

Angesichts der Machtverhältnisse der beiden Staaten ist es verständlich, daß Burmas Außenpolitik darauf zielt, den chinesischen Drachen möglichst zu besänftigen. Burmas Innenpolitik, vor allem seine Einstellung zu den einheimischen Kommunisten, bleibt unabhängig. Nach 1964 gingen die „Weißen Fahnen“, burmesische Kommunisten, zu einer revolutionär-maoistischen Linie über; ihre Führer waren sogar in Peking ausgebildet. China war laut Gurtov bereit, die Rebellen zu unterstützen, da zwischen China und Burma Differenzen aus verschiedenen Gründen auftraten, aufgrund des Schweigens zum Vietnam-Konflikt, aufgrund zunehmender Kontakte Burmas zu westlichen Ländern und über Burmas Einschränkung der politischen Tätigkeit der chinesischen Minderheit. Der Bruch erfolgte Juli 1967 nach schweren anti-chinesischen Unruhen in Rangoon. Die Pekinger Volkszeitung (Jen-min Jihpao) begrüßte darauf die „national-demokratische" Revolution der KP Burmas und attackierte die Regierung $\mathrm{Ne}$ Wins als reaktionär und faschistisch. Im Laufe der folgenden drei Jahre, da Burma nicht vom Neutralismus abwich, kehrte China allmählich zu freundschaftlicheren Beziehungen zu Burma zurück.

Gurtov betrachtet die neutralistische Außenpolitik Burmas und Kambodschas (unter Sihanouk) als gelungen und lobt die Fähigkeit dieser Länder, ihren territorialen Bestand, ihre staatliche Unabhängigkeit und Entscheidungsfreiheit gegenüber China zu behaupten. Er glaubt, daß China von 1965-1970 gleichzeitig gute Beziehungen $\mathrm{zu}$ neutralistischen Ländern pflegte und Rebellionen gegen unfreundliche Regierungen unterstützte. Diese Politik wurde in erster Linie durch die Ereignisse in Südostasien (nicht zuletzt in Vietnam) und weniger von ideologischen Grundsätzen gesteuert. Die Periode der Kulturrevolution August 1966-67 muß allerdings ausgeklammert werden. Weder sei Südostasien ein Machtvakuum, noch sei das Gebiet für eine amerikanische Politik eines Gleichgewichts der Mächte geeignet. Vielmehr werde dort ein kräftemäßiges Ungleichgewicht herrschen, innerhalb dessen die kleinen südostasiatischen Staaten eine relativ große Bewegungsfreiheit gegenüber China würden behaupten können.

Fünf Jahre bei der Rand Corporation tätig und der chinesischen Sprache kundig, liest Gurtov zwischen den Zeilen der Volkszeitung und der Roten Fahne. Er analysiert die kurzgefaßten Sendungen Radio Pekings und notiert, wer wen besucht. Im Gegensatz zu manchem „Pekingologen“ versucht er aber nicht nur, Pekings Äußerungen zu deuten, sondern sein Handeln zu werten. Man sollte jedoch nicht übersehen, daß diese Studie zum Teil auch einer heute gängigen Richtung folgt. Während vor einigen Jahren im Kreise der Fachwelt über Pekings Hilfe (und sei sie nur verbal) für Rebellionen in Südostasien Empörung herrschte, ist Gurtov der Überzeugung, daß China die Unabhängigkeit der südostasiatischen Länder nicht bedrohen würde, wenn Amerika sich aus Südostasien zurückziehen sollte. Ist hier 
die Vermutung berechtigt, daß Auswahl und Analyse der Daten von dem gewünschten Schluß beeinflußt sind, in diesem Falle dem, daß Chinas Außenpolitik sich defensiv verhalte? Dadurch, daß Gurtov immer wieder auf die südostasiatischen Gegenspieler Chinas weist, bringt er trotzdem eine informative Studie sowohl für China-Kenner wie auch für jene, die hauptsächlich an der Außenpolitik der südostasiatischen Länder interessiert sind.

Mary F. Somers Heidhues

\section{NORbert Horn}

\section{Das Recht der internationalen Anleihen}

Reihe Wirtschaftsrecht und Wirtschaftspolitik Band 27

Athenäum Verlag, Frankfurt/Main 1972

In einer sich ständig weiter verflechtenden internationalen Wirtschaft hat in den letzten Jahren der wachsende Finanzbedarf gerade auch in Europa zur Herausbildung eines eigenständigen internationalen Kapitalmarktes geführt. Begriffe wie Euro-Emissionen und Eurodollarmarkt sind dem Leser des Wirtschaftsteiles der Zeitung heute geläufig, ohne daß er jedoch immer spezifische Vorstellungen mit diesen Begriffen verbindet. Schlagzeilen über den Euro-Anleihenmarkt gab es im Jahre 1970, als es erstmalig zu Zahlungsschwierigkeiten und Zusammenbrüchen von Anleiheschuldnern kam.

Das vorliegende Werk, eine Frankfurter Habilitationsschrift aus dem Jahre 1971, ist die erste umfassende juristische Untersuchung des Rechts der internationalen Anleihen. Der Verfasser hat zur Bewältigung dieses komplexen Themas keine Mühen gescheut, um auch das meist sehr schwer zugängliche Material an den internationalen Finanzplätzen zu sammeln und es in den USA anhand der entsprechenden Fachliteratur und in Gesprächen mit Experten zu analysieren. Die Arbeit schildert die Rechtsfragen des Euro-Emissionsmarktes, wobei auch auf die Rechtsbeziehungen der Emittenten, die Emissionstechnik und auf die wichtige Frage des Investorenschutzes eingegangen wird. Bei der Darstellung der Rechtsfragen des Marktes geht Horn detailliert auf die rechtliche Regelung des Kapitalverkehrs, die "securities regulations“ und die steuer- und wirtschaftslenkungsrechtlichen Aspekte und Probleme des Euro-Emissionsmarktes ein. Die Rechtsstellung der Emittenten, d. h., derjenigen, die Teilschuldverschreibungen einer Anleihe ausstellen und sich in diesen als Hauptschuldner verpflichten, wird anhand der Praxis und unter besonderer Berücksichtigung der Rolle der Finanztochtergesellschaften dargestellt. Es schließt sich an eine Untersuchung der verschiedenen Emissionsverträge, die zur Erstausgabe von Kapitalmarktpapieren geschlossen werden: Übernahmevertrag, Emissionskonsortialvertrag und Distributionsvertrag werden ausführlich analysiert. Ein weiteres Kapitel widmet Horn der Rechtsstellung der Anlagegeläubiger. Im 4. Kapitel geht Horn schließlich auf die interessante Organisation des Anleihedienstes ein. Als wichtiges Beispiel der Entstehung eines einheitlichen Grundtypus schildert Horn den dem Common Law enstammenden Anleihetrust und seine kontinental-europäischen Abwandlungen. Er weist nach, daß alle für internationale Anleihen verwendeten Anleihetrusts nicht nur in vielen Details ähnliche Regelungen aufweisen, sondern auch in der zentralen Regelung, der Bestimmung und Funktion des Anleihetrustees, übereinstimmen. Horn kommt dann auf den außerordentlich vielschichtigen Problemkreis der Ausübung und des 